\title{
Las escuelas al aire libre uruguayas: creación y circulación de saberes
}

Uruguayan open-air schools: creation and circulation of knoweledge

André Dalben

Universidade Estadual de Londrina, Brasil

\section{ReSUMEN:}

El objetivo es analizar la creación de las primeras escuelas al aire libre en Uruguay y la posición asumida por el médico Américo Mola en la circulación nacional e internacional de saberes sobre estas instituciones médico-educativas. Las fuentes empleadas abarcan principalmente el periodo entre los años de 1909 y 1936. La primera escuela al aire libre fue inaugurada en Montevideo en 1913 por iniciativa de la Liga Uruguaya contra la Tuberculosis. Su funcionamiento contaba con la colaboración del Cuerpo Médico Escolar y de la Dirección General de Instrucción Primaria del Uruguay. A principios de la década de 1920, el gobierno uruguayo creó otras dos escuelas al aire libre en los alrededores de la capital uruguaya. Mola fue una figura central para la divulgación internacional de las tres escuelas al aire libre uruguayas. En 1922 asumió la presidencia del Comité Internacional de Escuelas al Aire Libre y en 1936 presidió la tercera edición del Congreso Internacional de Escuelas al Aire Libre (Bielefeld/Alemania). A lo largo de su carrera estableció una fructífera red de interlocución con profesionales sudamericanos y europeos por medio del Instituto Internacional Americano de Protección a la Infancia para la divulgación internacional de las escuelas al aire libre uruguayas.

Palabras Clave: Educación al aire libre, Escuelas al aire libre, Historia, Uruguay.

\section{Abstract:}

The purpose of this article is to analyze the creation of the first open-air schools in Uruguay and the position taken by the physician Américo Mola in both the national and international circulation of knowledge about these medical-educational institutions. The sources used in this research cover mainly the period between the years 1909 and 1936. The Uruguayan League against Tuberculosis inaugurated the first open-air school in Montevideo in 1913. It operated in collaboration with the School Medical Staff and the General Administration of Primary Education of Uruguay. In the early 1920s, the Uruguayan government created two other open-air schools in the vicinity of the capital. Mola was a key figure for the international promotion of the three Uruguayan openair schools. In 1922, he assumed the presidency of the International Committee of Open-Air Schools and in 1936 presided the third edition of the International Congress of Open-Air Schools (Bielefeld/Germany). Throughout his career, he established a fruitful network of contacts with South American and European professionals through the American International Institute for Child Protection, in order to promote Uruguayan open-air schools in the international scenario.

KEYWORDS: Outdoor Education, Open-Air Schools, History, Uruguay.

\section{INTRODUCCIÓN}

Las escuelas al aire libre son un tema de investigación muy reciente en la historia de la educación física y de la educación. Se tratan de instituciones que pueden ser definidas como un "cometa médico-pedagógico" que pasó de manera fugaz por distintos países dejando pocas huellas en la memoria colectiva, o mismo en las investigaciones historiográficas. Nacidas en la aurora del siglo XX y abiertas en diferentes países, son raras las escuelas al aire libre aun en actividad en el mundo, pues la gran mayoría desapareció en los años 1950 (Luc, 2003).

La definición de un "cometa médico-pedagógico" apunta no solo a la brevedad histórica de estas instituciones, sino también a la ambición de asociar la educación con una política médica de prevención de la tuberculosis, enfermedad que afecta a muchos países a principios del siglo XX. Las escuelas al aire libre tienen sus raíces médicas en antiguos saberes de la medicina natural. Esta vertiente médica, desarrollada a lo largo del siglo XIX, fue responsable por crear métodos de curación y prevención que utilizaban la acción terapéutica de los elementos naturales - como el agua, la luz del sol, el aire de altitud, los ejercicios físicos al aire libre y los alimentos. Estos eran recursos adoptados para combatir la expansión de la tuberculosis, en un momento en que los medicamentos alopáticos todavía estaban en desarrollo. Los tratamientos de 
hidroterapia, helioterapia y aeroterapia, como se denominaron, ganaron gran aprecio por parte de los médicos en fines del siglo XIX, y fueron empleados inicialmente en establecimientos de curación - como los sanatorios infantiles para tratar la tuberculosis, o de prevención - como las colonias de vacaciones (Armus, 2007; Baubérot, 2004; Dalben, 2015; Villaret, 2005; Villaret \& Saint-Martin, 2004).

Las escuelas al aire libre surgieron como respuesta a una serie de críticas que comenzaron a emerger, en la transición del siglo XIX al siglo XX, contra los sanatorios infantiles y las colonias de vacaciones. En este momento, muchos profesionales pasaron a considerar el período de vacaciones escolares muy corto para garantizar efectivos beneficios a la salud de los niños atendidos en las colonias de vacaciones ubicadas en las playas, campos y montañas. Además, al regresar de los viajes, los niños atendidos continuarían viviendo bajo precarias situaciones de saneamiento y vivienda, lo que podría ponerlos nuevamente en riesgo de contagio de la tuberculosis. Otra cuestión fue el hecho de los sanatorios infantiles promover un aislamiento de los niños de la educación formal cuando el tratamiento médico se prolongaba, acarreando retraso escolar. En contrapartida, las escuelas al aire libre podrían permitir que los niños atendidos fuesen sometidos a las terapias naturales y a los ejercicios físicos al aire libre a lo largo de meses sin que el proceso de escolarización fuese interrumpido (Ludwing, 2003).

Las primeras escuelas al aire libre nacieron concomitantemente en Alemania y Bélgica en el año de 1904. Estas primeras experiencias han encontrado un proficuo eje de difusión en los Congresos Internacionales de Tuberculosis $(1905,1906,1908,1912)$ y en los Congresos Internacionales de Higiene Escolar (1904, 1910, 1913) (Châtelet, 2003). A lo largo del tiempo, la circulación de saberes promovida por los congresos posibilitó que varios otros países creasen sus propias experiencias de escuelas al aire libre. En 1922 ocurrió el primer Congreso Internacional de Escuelas al Aire Libre (París), evento que buscó establecer líneas directrices para intentar unificar un movimiento internacional polisémico que se encontraba en plena expansión (Jablonka, 2003). En 1922 fue también creado el Comité Internacional de las Escuelas al Aire Libre, bajo la presidencia del médico uruguayo Américo Mola, lo que llevó a la investigadora Anne Marie-Châtelet (2003) a deducir que el movimiento también se extendió a América del Sur.

Para la historia de la educación física, las escuelas al aire libre corresponden a un proficuo tema de investigaciones, una vez que los ejercicios físicos al aire libre, como la gimnasia respiratoria y los juegos en la naturaleza, ganaron especial aprecio y centralidad en los cronogramas de estas instituciones médicoeducativas (Dalben, 2014). Los ejercicios físicos al aire libre fueron interpretados sobre todo como fuentes de regeneración de las energías físicas de los niños, siendo empleados para el robustecimiento de sus cuerpos y para la prevención de enfermedades. En las investigaciones históricas de la educación física, se puede localizar las escuelas al aire libre como parte de un movimiento cultural mayor relacionado al advenimiento de la vida al aire libre a partir de la expansión de las ciudades, y que buscó promover diferentes medios para asegurar el contacto del cuerpo con los elementos de la naturaleza (Andrieu, 2011, 2017; Rauch, 1983; Sirost, 2009; Soares, 2016).

\section{ОвJеtivo}

El objetivo de la investigación es analizar la creación de las primeras escuelas al aire libre en Uruguay y la posición asumida por el médico Américo Mola en la circulación nacional e internacional de saberes sobre estas instituciones médico-educativas. Cabe advertir que en lugar de crear o reforzar un mito heroico y patriótico para la figura de Mola, se buscó rescatar la participación de este médico uruguayo en los debates nacionales e internacionales sobre las escuelas al aire libre y analizar sus estrategias para establecer una red de sociabilidad con profesionales de diferentes países. 


\section{Metodología}

La investigación empleó un conjunto de fuentes documentales sobre las escuelas al aire libre uruguayas disponibles en: a) Biblioteca Nacional de Uruguay; b) Biblioteca Pedagógica Central "Mtro. Sebastián Morey Otero" del Museo Pedagógico en Montevideo; c) Biblioteca Especializada “Dr. Luis Morquio” del Instituto Interamericano del Niño, la Niña y Adolescentes en Montevideo; d) Biblioteca Nacional de Maestros en Buenos Aires. Las fuentes se constituyeron de: a) periódico Anales de Instrucción Primaria; b) Enciclopedia de Educación, ambos publicados por la Dirección General de Instrucción Primaria de Uruguay; c) Memorias del Cuerpo Médico Escolar de Uruguay; d) Acta del III Congreso Internacional de Higiene Escolar; e) Actas de los Congresos Americanos del Niño; f) Boletines del Instituto Internacional Americano de Protección a la Infancia; g) ediciones de la Revista Médica del Uruguay; h) y libros. Las fuentes abarcan principalmente el periodo que comprende los años de 1909 hasta 1936, desde el momento que se comenzó a sugerir la creación de escuelas al aire libre en Uruguay hasta el año en que el médico Américo Mola presidió el III Congreso Internacional de Escuelas al Aire Libre, ocurrido en la ciudad de Bielefeld en Alemania.

\section{Resultado y Discusión}

\section{Los primeros proyectos de prácticas e instituciones al aire libre en Uruguay}

En agosto de 1908 fue creado el Cuerpo Médico Escolar (CME). Sus cuatro médicos inspectores eran encargados de fiscalizar la higiene de las escuelas y asesorar a la Dirección General de Instrucción Primaria (DGIP) en medidas a ser tomadas e instituciones a ser creadas para garantizar la salud de los estudiantes y del personal enseñante (Schiaffino, 1929). Estos médicos también formaban parte de la Comisión Honoraria de Prevención Escolar contra la Tuberculosis, encargada de encontrar soluciones para la epidemia de tuberculosis que afectaba al país (Rodríguez, 1909).

En abril de 1909, Sebastián R. Rodríguez, uno de los médicos del CME, propuso a la DGIP un proyecto para la organización de paseos o excursiones escolares todos los jueves del año escolar. Su intención era aliar la instrucción a los preceptos de medicina natural. La propuesta era que, durante los paseos a lugares como las quintas, parques u orillas del mar, los profesores ministrasen lecciones recreativas e instructivas sobre historia o ciencias naturales. "Se insistió constantemente en los beneficios higiénicos y pedagógicos de trabajar al aire libre, en 'contacto con la naturaleza"' (Espiga, 2015, p.145). Como justificación Rodríguez consideraba más viable y más factible, por el momento en Uruguay, la organización de paseos o excursiones escolares en lugar de la creación de colonias de vacaciones (Rodríguez, 1909).

Sin embargo, los profesionales del CME no excluyeron la posibilidad de envío de los escolares de la capital uruguaya para la colonia de vacaciones ya existente en Punta Carretas. Esta colonia era organizada por la Comisión Nacional de Caridad y Beneficencia Pública de Uruguay ${ }^{1}$ especialmente para los niños de sus orfanatos. En septiembre de 1909 establecieron una alianza con la comisión para admisión en la colonia de alumnos de las escuelas públicas. Setenta y siete niños fueron seleccionados por los médicos del CME, pero apenas diez siguieron en viaje debido a la oposición manifestada por los padres. Como consecuencia, diría uno de los profesionales del CME, "las autoridades encargadas de velar por la instrucción y la salud de la población infantil, no han de dilatar la fundación de otras Colonias Escolares" (Cuerpo, 1910, p.152).

En el mismo periodo, el Inspector de Instrucción Primaria de Montevideo, Eduardo Rogé (1862-1938), divulgaba en los Anales de Instrucción Primaria las iniciativas de escuelas al aire libre llevadas a cabo en Argentina $^{2}$ y en diferentes países europeos, como Alemania, Francia e Inglaterra. Llamaba la atención sobretodo sobre las diferencias entre las colonias de vacaciones y las escuelas al aire libre. Informaba que mientras las colonias funcionaban solamente en los meses de vacaciones escolares y no ofrecían clases, las 
escuelas al aire libre podrían quedar abiertas por un periodo significativamente más largo de meses y ofrecerían clases de acuerdo con los programas de la escuela primaria común. En diciembre de 1909 presentó a la DGIP un proyecto para la creación de una escuela al aire libre en Montevideo. Su propuesta era de crear una escuela al aire libre empleando la estructura de un chalet que servía entonces como escuela rural en la localidad del Cerrito de la Victoria, pero que podría ser desmontado y armado nuevamente en el barrio del Prado, en las proximidades del paseo público (Rogé, 1910). El proyecto, no obstante, no fue llevado a cabo.

\section{La creación de las primeras escuelas al aire libre uruguayas (1913-1927)}

En 16 de junio de 1913 empezó a funcionar la primera escuela al aire libre de Montevideo, fundada por iniciativa de la Liga Uruguaya contra la Tuberculosis ${ }^{3}$ (LUT) e instalada en su parque en el barrio Larrañaga. Para su funcionamiento contaba con la colaboración del CME y de la DGIP. "El objetivo fue 'robustecer a los niños débiles y propensos a la tuberculosis jerarquizando la actividad física o 'gimnasia respiratoria”" (Espiga, 2015, p.143). Comenzó sus clases con una inscripción de 33 niños, nmero que llegó a 99 en diciembre. El exponencial aumento de inscripciones fue considerado como prueba de "la aceptación que por parte de los padres va teniendo esta escuela, cosa que no sucedió con la Colonia de Vacaciones instalada en Punta Carretas en 1909, donde tal vez por ignorancia hubo cierta resistencia para enviar a los niños" (Rodríguez, 1914, p. 26). La gran adhesión que tuvo la escuela fue interpretada por los profesionales del CME por el hecho de que los niños regresaban todas las tardes a sus casas, lo que garantizaría la conservación de un vínculo más estrecho con sus familias. Consideraban que el aumento de inscripciones era prueba de "las ventajas que ha puesto en evidencia la práctica del funcionamiento de la Escuela al Aire Libre sobre las colonias de vacaciones, creemos que son dignas de tenerse en cuenta y deben alentar a las autoridades escolares a aumentar su número('Schiaffino, 1914, p. 92). La escuela, dirigida por Pilar Llanche, era hasta entonces la únical aire libre en Uruguay (Llanche, 1919).

En el año siguiente el médico Rafael Schiaffino, profesional del CME, presentó proyecto a DGIP para la creación de una "escuela de la playa" para permanencia de los niños durante el verano. Se preveía su instalación en alguna playa adjunta a la ciudad de Montevideo, como Ramírez, Pocitos o Capurro, de modo que los niños pudiesen regresar a sus casas todos los días. La institución podría ser, en las palabras de su proponente, "el complemento de las Escuelas al Aire Libre en el campo" (Schiaffino, 1914, p.94). El proyecto fue presentado también en la segunda edición del Congreso Panamericano del Niño, ocurrido en Montevideo en el año de 1919, pero no fue llevado a cabo en aquel momento (Schiaffino, 1929). ${ }^{4}$

En 1918, el CME recomendó nuevamente que las escuelas den sus lecciones fuera de los salones, como había anunciado Rodríguez en 1909 (Espiga, 2015). En este momento, las clases e instituciones de enseñanza al aire libre empiezan a mostrarse como una alternativa plausible y relevante entre los médicos y maestros uruguayos. A principios de la década de 1920 dos escuelas al aire libre fueron creadas por el gobierno uruguayo en los alrededores de la capital, distantes de las aglomeraciones y de las fábricas, pero servidas por tranvía y ubicadas en parques propios. La última escuela creada estaba situada en un parque especialmente grande, de más de siete hectáreas, con árboles frondosos y muchas plantas. En 1927 la primera escuela al aire libre creada en Montevideo se trasladó a un parque proprio, desvinculándose por completo de la LUT (Mola, 1927). A pesar de instaladas en parques propios, ninguna se encontraba en un lugar con construcciones expresamente planificadas para su funcionamiento en aquel momento. Las tres escuelas eran mantenidas enteramente por el presupuesto nacional, siendo administradas por profesionales de la DGIP e inspeccionadas por el médico Américo Mola 5 (Fournié, 1928).

Aun en el año 1927 otra escuela al aire libre fue inaugurada en Tacuarembó por iniciativa común entre la Dirección de Instrucción Primaria del Departamento y la LUT. Según su directora, la institución seguía 
los mismos parámetros médicos y pedagógicos instituidos para las escuelas congéneres de Montevideo (Fernández, 1927).

\section{El cotidiano de las tres escuelas al aire libre de Montevideo}

Las tres escuelas al aire libre creadas en Montevideo eran mixtas y tenían la capacidad de atender cerca de 350 niños y niñas de 6 a 12 años de edad, desde las 9 hasta las 17 horas en verano y desde las 10 hasta las 16 horas en invierno. Los niños evaluados por los médicos inspectores del CME como físicamente más débiles (desnutridos) o considerados como predispuestos a enfermedades, como la tuberculosis, eran seleccionados en las escuelas de la red de enseñanza pblica y permanecían en las escuelas al aire libre por un periodo mínimo de cuatro meses. Cada año las escuelas llegaban a recibir a cerca de 1500 niños, en su gran mayoría hijos de familias pobres. La programación médica dirigida por Mola incluía sesiones diarias de helioterapia, adaptadas del método creado por el medico suizo Auguste Rollier. ${ }^{6}$ A cada semana era mensurado el peso de los niños y trimestralmente sus talla y perímetro torácico. Los alimentos ofrecidos en desayuno, almuerzo y merienda eran especialmente planificados por los médicos. Al final de cuatro meses la gran mayoría de los niños regresaba a sus escuelas de origen (Mola, 1927, 1930a, 1930b, 1936).

\section{FIGURA 1}

Escuela al Aire Libre no 3 - Clase al aire libre en verano

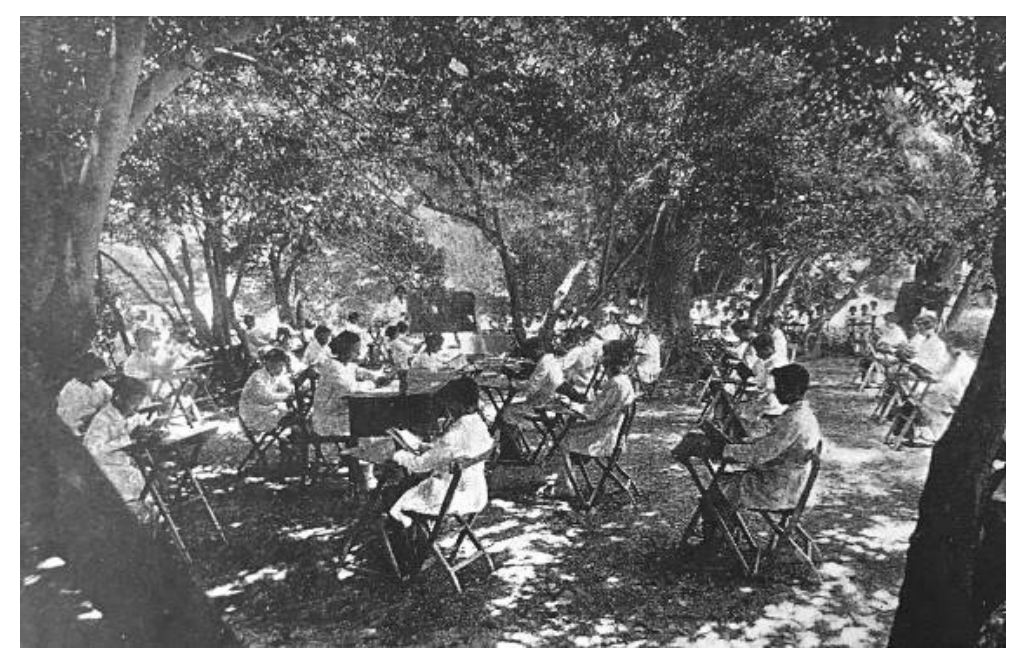

(Mola, 1927, p. 207) 
FIGURA 2

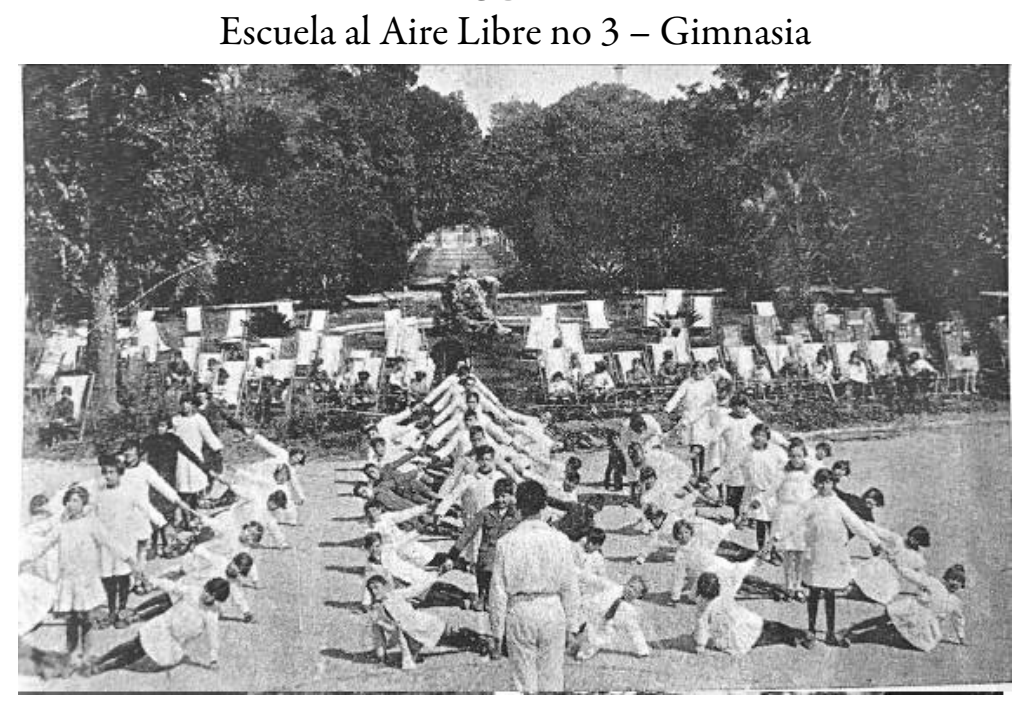

(Fournié, 1927, p. 263)

En las tres escuelas se utilizaban pupitres livianos y plegadizos para las clases, dispuestos en distintos lugares de los parques (Figura 1). De acuerdo con su inspector técnico de enseñanza, Emílio Fournié (1927, p. 254), "al ingresar un grupo de niños, el maestro de cada clase hará un examen escrito sobre las materias fundamentales, a fin de apreciar el término medio de conocimientos de sus nuevos alumnos". El tiempo de las clases era reducido, con una duración total de dos horas y media en el programa escolar, siendo intercaladas por la helioterapia, gimnasia respiratoria, juegos dirigidos, trabajos manuales, trabajos de huerta y jardinería, canto y la siesta después del almuerzo (Figura 2). El contenido de las clases de matemática, lenguaje, higiene y moral, y ciencias naturales era lo mismo de las escuelas comunes, con la diferencia que toda la enseñanza tenía "un carácter esencialmente práctico, aprovechando la circunstancia de funcionar la escuela en un parque y al aire libre" (Fournié, 1927, p. 254).

Los métodos activos de enseñanza eran priorizados en las clases y la enseñanza formal, debidamente planificada y estructurada, se complementaba por la enseñanza informal u ocasional - derivada de cualquier incidencia cotidiana o de las demás actividades previstas en el programa escolar. La intención era hacer la enseñanza "no solamente a la hora de la clase propiamente dicha, sino durante todo el día" (Fournié, 1927, p. 258), compensando el tiempo reducido de las clases. Todo el programa escolar y los métodos de enseñanza estaban pensados para que no hubiese retraso escolar, aunque con menos horas de clase, pudiendo el niño continuar normalmente los estudios al regresar a su escuela de origen.

\section{La circulación de saberes acerca de las escuelas al aire libre uruguayas (1927-1936)}

Como hemos visto, Eduardo Rogé fue el responsable de divulgar en Uruguay las experiencias de escuelas al aire libre que se estaban realizando en el exterior a principios del siglo XX (Rogé, 1910). Estos conocimientos ciertamente fueron apropiados para la creación de las primeras escuelas al aire libre uruguayas. La proximidad con Argentina, país que había creado sus escuelas al aire libre pocos meses antes también debe haber ejercido cierta influencia. A partir de la década de 1920, el Uruguay se convertiría, sin embargo, en un centro de divulgación internacional de saberes sobre las escuelas al aire libre. Las experiencias desarrolladas en sus tres escuelas de Montevideo ganaron proyección internacional con el trabajo sistemático de divulgación que sería realizado por el médico Mola (profesional del CME) y por el inspector técnico de enseñanza, Fournié, en los Boletines del Instituto Internacional Americano de Protección a la Infancia (BIIAPI). ${ }^{7}$ 
Las primeras publicaciones de los dos profesionales, realizadas en 1927, presentaron de forma detallada la organización médica y pedagógica de los establecimientos uruguayos. Mola (1927) declaraba en su texto que las escuelas creadas en Uruguay seguían las definiciones instituidas en el primer Congreso Internacional de Escuelas al Aire Libre, celebrado en 1922 en Francia. En el mismo año Mola había sido elegido como presidente del Comité Internacional de Escuelas al Aire Libre, comisión responsable de reunir informaciones y divulgar las experiencias de escuelas al aire libre (Châtelet, 2003, p.26). No por casualidad, en la misma edición del BIIAPI, Fournié (1927) solicitaba en su artículo que los demás países americanos enviasen informes sobre posibles experiencias de escuelas al aire libre puestas en práctica en sus territorios.

En el año siguiente, Fournié (1928) volvió a escribir en el boletín, en respuesta a los pedidos provenientes de Colombia y Chile de mayores informaciones sobre el funcionamiento de las escuelas al aire libre. Además de las mayores especificaciones sobre las escuelas uruguayas, brindó también una amplia bibliografía internacional de artículos académicos y de la prensa diaria sobre las escuelas al aire libre que se encontraban bajo la custodia del Instituto Internacional Americano de Protección a la Infancia (IIAPI). Fournié también solicitaba a aquellos que conociesen otras publicaciones para enviarlas al Instituto. El tema se convertía, así, de gran interés no sólo para los dos profesionales como para el propio IIAPI, tornándose en uno de los principales responsables por registrar y divulgar las escuelas al aire libre puestas en práctica en diferentes países americanos.

En el boletín Mola también relató su participación en el Congreso Internacional de Protección a la Infancia, celebrado en 1928 en París (Mola, 1930a). Se trataba de un evento de grandes proporciones, que contó con la participación de más de 800 delegados de 39 países, que discutieron temas como legislaciones, salud, asistencia y protección a la infancia. El congreso formaba parte de la Quincena Social de París, evento que reunía otros tres congresos y una Exposición Internacional, siendo el de protección a la infancia presidida por el médico uruguayo Luis Morquio. ${ }^{8} \mathrm{El}$ asunto de las escuelas al aire libre fue, en realidad, el tema de destaque de la delegación uruguaya, siendo anunciado por Morquio ya en la ceremonia de apertura del congreso (Mariano y Mariano, 2016). En el viaje a Europa por la ocasión del congreso, Mola aprovechó aun para establecer intercambios internacionales y conocer algunas de las escuelas al aire libre existentes en Francia y Bélgica, así como los establecimientos mantenidos por Rollier en Suiza. En la Exposición Internacional adjunta a los congresos presentó una serie de fotografías de las tres escuelas al aire libre de Montevideo, alcanzando gran notoriedad entre los participantes (Mola, 1930a). Al mismo tiempo que Mola se especializaba en el eje temático de las escuelas al aire libre dentro del área de la asistencia social infantil, también obtenía reconocido prestigio internacional.

En el ámbito nacional, a lo largo de su carrera Mola escribió informes relatando el funcionamiento de las tres escuelas de Montevideo y sus participaciones en eventos internacionales (Mola, 1936). Para garantizar la amplia circulación de sus textos, los reprodujo en revistas de medicina, en los Anales de Instrucción Primaria, en la Enciclopedia de Educación y en formato de libreto (Mola, 1917, 1923, 1928, 1930b, 1940). Es cierto que la importante diversidad de periódicos elegidos por Mola para diseminar sus textos ayudó a que alcanzasen una gran circulación entre la comunidad científica uruguaya. Mola sería reconocido como una autoridad médica que se dedicaba "con verdadero cariño y competencia a los niños débiles, trayendo, además, ahora interesantes proyectos e innovaciones de Europa” (Dirección de Enseñanza Primaria y Normal, 1929, p.160). Mola no fue el único profesional uruguayo a defender las escuelas al aire libre o dedicarse a la temática de la educación al aire libre en Uruguay. ${ }^{9}$ Sin embargo, se puede afirmar que la estrategia de construcción de su memoria profesional fue muy bien articulada por él mismo, por medio de la serie de publicaciones que realizó a nivel nacional e internacional y de la red de interlocución que construyó con profesionales locales y extranjeros, de modo que luego se transformaría en un nombre casi inevitable cuando se trataba de debatir las escuelas al aire libre en Uruguay.

En 1931, Mola viajó nuevamente a Europa. Esta vez para participar de la segunda edición del Congreso Internacional de Escuelas al Aire Libre, ocurrido en Bruselas, y de la Conferencia Internacional de Colonias 
de Vacaciones y Obras al Aire Libre, ocurrida en Ginebra (Turnes, 2015). Seguramente estas también fueran ocasiones para encontrarse con los demás profesionales que componían el Comité Internacional de Escuelas al Aire Libre, el médico alemán Karl Triebold (vicepresidente) y el médico francés Gaston Lemonier (secretario general), y estrechar sus vínculos profesionales.

En el año de 1935 el gobierno uruguayo empezó la expansión de las escuelas al aire libre en su territorio, con la organización de un sistema nacional que abarcaría prácticamente todos los departamentos del interior del país. Se inauguraron escuelas al aire libre primero en las capitales de los departamentos de Paysandú, Rocha y Salto. En el año siguiente fue la vez de los departamentos de Artigas, Canelones, Durazno, Flores y Treinta y Tres, y en 1937 todos los demás, con excepción de Florida. En todas las capitales de departamento prácticamente fue inaugurada una escuela al aire libre (Friedrich, 1940). Es probable que Mola no haya sido el responsable directo o el coordinador de esta expansión, una vez que ningún documento analizado afirma esta hipótesis. En realidad, la DGIP buscaba, desde 1929, terrenos en cada uno de los departamentos uruguayos para expandir el proyecto de Eduardo Jiménez de Aréchaga, Emilio Oribe y Emilio Verdesio de creación de instituciones infantiles al aire libre (Dirección, 1929). Es posible, así, que hubiese embates y divergencias entre diferentes grupos profesionales y sus respectivos proyectos de instituciones al aire libre a nivel nacional. Además, las propuestas de Mola no siempre eran aceptadas por la DGIP. A pesar de protestar, por ejemplo, que la dirección de las escuelas al aire libre de Montevideo debía ser pasada a los médicos, otorgándoles mayor autoridad y ampliando la dependencia y vigilancia médica en los establecimientos existentes en Montevideo, esta reivindicación no fue atendida en aquel momento (Mola, 1930a, 1936).

Sin embargo, como se ha analizado anteriormente, Mola consiguió afirmarse como una de las principales voces nacionales sobre el tema de las escuelas al aire libre y estableció un considerable intercambio de saberes entre su país de origen y los países americanos y europeos. Su consagración internacional ocurrió en 1936, cuando fue invitado para presidir la tercera edición del Congreso Internacional de Escuelas al Aire Libre. En sus palabras:

En mi carácter de Delegado oficial del Gobierno del Uruguay, y representando al Consejo del Niño y al Consejo de Enseñanza Primaria y Normal, invitado oficialmente por el Gobierno alemán, tuve el honor de presidir el Tercer Congreso Internacional de Escuelas al Aire Libre que se realizó en Bielefeld (Alemania) (Mola, 1936, p.404).

El congreso, ocurrido entre 18 y 23 de julio de 1936, fue, en realidad, completamente planeado y organizado por el gobierno alemán, responsable también por invitar a los veintiséis países ${ }^{10}$ que se hicieron presentes. Participaron alrededor de 400 profesionales extranjeros, número pequeño frente a los miles de profesionales alemanes presentes. La mayor parte de la programación del evento fue destinada a actividades culturales, presentaciones artísticas, excursiones y visitas a instituciones y organizaciones educativas alemanas (Brun, 1936). La organización de su programación demuestra el intento de Alemania de propagar y consagrar las organizaciones nazistas de educación al aire libre, como la Juventud Hitleriana, frente a los demás países presentes. La fecha de su realización tampoco fue ocasional, pocos días antes de la celebración de los Juegos Olímpicos de Berlín, lo cual buscó mostrar al mundo el poderío de Alemania nazista (Cubillas, 2011). La invitación hecha a Mola para presidir el congreso se configuraba como una estrategia de legitimación del evento ante la comunidad científica internacional, una vez que el médico uruguayo era el representante máximo del Comité Internacional de Escuelas al Aire Libre. En sus relatos sobre el congreso, hecho en el BIIAPI, Mola poco escribió sobre los debates ocurridos, atendiendo sobre todo a las presentaciones hechas por las autoridades alemanas de sus organizaciones de educación al aire libre. En la ocasión del congreso, Mola fue reelegido para el cargo de presidente del Comité Internacional de Escuelas al Aire Libre (Mola, 1936). 


\section{CoNCLUSIón}

Las primeras escuelas al aire libre creadas en Montevideo se establecieron como una alternativa para vencer la baja adhesión que tuvo la primera colonia de vacaciones destinada a los niños de las escuelas públicas. La resistencia de los padres puede estar relacionada con factores diversos, como el desconocimiento de qué sería aquella iniciativa aún poco usual en la época; el temor de que sus hijos fuesen estigmatizados socialmente como enfermos, una vez que la colonia de vacaciones podría ser confundida con sanatorios para tuberculosos, o debido al término niño débil a menudo empleado en la época; el aislamiento de la convivencia familiar promovido por la colonia resultante de su formato de internado temporario; o hasta la inexistencia de tiempo libre por cuenta del trabajo infantil, una vez que se trataban de familias en situación de pobreza. En razón de la gran adhesión a la primera escuela al aire libre, este modelo de institución médico-educativa fue considerado como más ventajoso y la DGIP priorizó invertir en la creación de nuevos establecimientos de este género en lugar de las colonias.

Las escuelas al aire libre también nacieron en Uruguay como una política que puede ser encuadrada en el marco de la medicalización y normalización de la infancia, promovido, por ejemplo, por medio de la institucionalización de la medicina escolar con la creación del CME en el país (Espiga, 2015). Américo Mola, por ejemplo, se hallaba ligado a los movimientos de la eugenesia preventiva uruguaya y exigía constantemente que la dirección de las escuelas al aire libre de Montevideo fuese transferida a los médicos. Sin embargo, no podemos despreciar la advertencia hecha por Châtelet (2003) de que las escuelas al aire libre también fueron un paso importante para garantizar el derecho de los niños a la salud. La experiencia de Uruguay, por ejemplo, fue tomada por el IIAPI como una política ejemplar de protección y asistencia a los niños en situación de pobreza. Además, las escuelas al aire libre se estableceran en muchos países como experiencias de modernización de las arquitecturas, contenidos, programas y métodos escolares (Martinez, 2000; Rodrigo y Lis; 1999; Rodríguez, 2015; Ruchat, 2003; Savoye, 2003). Esta era, por ejemplo, la intención de Fournié (1927), que creía que las escuelas al aire libre uruguayas podrían, en un futuro próximo, atender no solo a los niños designados como débiles, sino a todos en edad escolar, convirtiéndose en escuelas modelos y de aplicación de nuevas pedagogías activas. ${ }^{11}$

El Uruguay buscó ocupar una posición de vanguardia en los debates internacionales sobre la protección de la infancia al dedicarse al tema de las escuelas al aire libre, y sus tres instituciones creadas en Montevideo han alcanzado gran proyección en el Congreso Internacional de Protección a la Infancia, ocurrido en 1928 en París. Según Mola (1930), por ejemplo, en la ocasión las escuelas al aire libre fueron aclamadas por ser completamente financiadas por el estado uruguayo, algo aún inusual en otros países. Las escuelas al aire libre fueron, en realidad, elegidas por Uruguay como una estrategia para alzarlo en una posición destacada en el concierto de las naciones, siendo uno de los únicos países en crear un sistema nacional de escuelas al aire libre, con instituciones distribuidas por todo el territorio nacional. Las experiencias uruguayas y las redes de sociabilidad que Mola creó por medio del IIAPI le permitieron solidificar una destacada carrera internacional, habiendo permanecido más de una década en la presidencia del Comité Internacional de Escuelas al Aire Libre y siendo invitado a presidir la tercera edición del Congreso Internacional de Escuelas al Aire Libre. Se consolidó, así, como una figura central en la divulgación y en la circulación de los saberes sobre las escuelas al aire libre en Uruguay, América y Europa.

\section{REFERENCIAS BIBLIOGRÁFICAS}

Andrieu, B. (2011). L'écologie corporelle: bien-être et cosmose. Paris: L'Harmattan.

Andrieu, B. (2017). L'écologie corporelle: émersion vivante et techniques écologiques. Paris: L'Harmattan.

Armus, D. (2007). La ciudad impura. Salud, tuberculosis y cultura en Buenos Aires, 1870-1950. Buenos Aires: EDHASA. 
Baubérot, A. (2004). Histoire du naturisme: le mythe du retour a la nature. Rennes: Presses universitaires de Rennes.

Brun, L. (1936). Le Troisième Congrès International des Écoles de Plein Air. Le Langues Modernes, 34 (8/9), 466-472. Recuperado de: http://gallica.bnf.fr/ark:/12148/

Camarillo, N. F. (2014)."Niños sanos, naciones fuertes: los Congresos Panamericanos del Niño como modelos de modernización, higiene y educación, 1916-1942” (Maestria en Historia Internacional). Centro de Investigación y Docencia Económicas.

Carbonell, J. F. (1927). Parques escolares. Montevideo: Federación Magistral Uruguaya.

Châtelet, A. (2003). Le mouvement international des écoles de plein air. En A. Châtelet, D. Lerch y J. Luc (Ed.), L'école de plein air: une expérience pédagogique et architecturale dans l'Europe du XXe siècle (pp. 21-36). Paris: Éditions Recherches.

Cubillas, L. V.S. (2011). Nazismo y deporte. Los juegos olímpicos de Berlín en 1936. Citius, altius, fortius: humanismo, sociedad y deporte: investigaciones y ensayos, 4 (1), 73-106.

Cuerpo Médico Escolar Nacional. (1910). La bigiene escolar en la República Oriental del Uruguay: trabajo presentado al III Congreso Internacional de Higiene Escolar - Paris 1910. Montevideo: A. Barreiro y Ramos.

Dalben, A. (2014). Mais do que energia, uma aventura do corpo: as colônias de férias escolares na América do Sul (1882-1950). (Doctorado en Educación). Universidade Estadual de Campinas.

Dalben, A. (2015). Artes de curar: embates históricos entre as terapias naturais e os tratamentos alopáticos. En R. C. B. Barros \& L. Masini, (Ed.), Sociedade e Medicalização (pp. 81-94). Campinas: Pontes.

Dirección de Enseñanza Primaria y Normal (1929). La moderna orientación de la escuela uruguaya: la Escuela Marítima. Anales de Instrucción Primaria, 26 (2), 146-204.

Espiga, S. (2015). La infancia normalizada: libros, maestros e higienistas en la escuela pública uruguaya 1885-1918. Montevideo: Antítesis.

Fernández, G. G. (1927). La escuela al aire libre que dirijo: su organización y plan de trabajos. Anales de Instrucción Primaria, 24 (2), 85-94.

Ferreira, C. V. (1927). Parques escolares. Montevideo: Editorial Guttenberg.

Fournie\#, E. (1927). Las escuela al aire libre, desde el punto de vista pedagógico. Boletín del Instituto Internacional Americano de Protección a la Infancia, 1 (2), 252-263.

Fournie\#, E. (1928). Consultas. Boletin del Instituto Internacional Americano de Protección a la Infancia, 1 (1), 119-128.

Friedrich, M. A. (1940). La enseñanza en las escuelas al aire libre. Enciclopedia de Educación, 3/2 (1), 219-224.

Jablonka, I. (2003). La réunion éphémère: les ambiguïtés du premier Congrès international des écoles de plein air (1922). En A. Châtelet, D. Lerch y J. Luc (Ed.), L'école de plein air: une expérience pédagogique et architecturale dans l'Europe du XXe siècle (pp. 7-20). Paris: Éditions Recherches.

Llanche, P. (1919). Escuelas al aire libre. En República Oriental del Uruguay. II Congreso Americano del Niño. (v.4 pp 95-107). Montevideo: Peña Hnos.

Luc, J. (2003). L'école de plein air : une histoire a découvrir. En A. Châtelet, D. Lerch y J. Luc (Ed.), L'école deplein air: une expérience pédagogique et architecturale dans l'Europe du XXe siècle (pp. 7-20). Paris: Éditions Recherches.

Ludwing, H. (2003). Les écoles de plein air en Allemagne, une forme de l'éducation nouvelle. En A. Châtelet, D. Lerch y J. Luc (Ed.), L'école de plein air: une expérience pédagogique et architecturale dans l'Europe du XXe siècle (pp. 39-55). Paris: Éditions Recherches.

Mariano, H. A. y Mariano, C. L. S. (2016). O debate sobre a infância na quinzena social de Paris de 1928 e o processo de criaçao do Instituto Interamericano del Niño. Projeto História, 55, 190-207.

Martinez, J. M. B. (2000). De las escuelas al aire libre a las aulas de la naturaleza. AREAS: Revista Internacional de Ciencias Sociales, 20, 171-182.

Martinis, P. (2013). Educación, pobreza y seguridad en el Uruguay de la década de los noventa. Montevideo: Universidad de la República.

Ministerio de Obras Públicas (1928). Parques escolares: contribución de la Dirección de Arquitectura al III Congreso Pan-Americano de Arquitectos. Montevideo: Ministerio de Obras Públicas. 
Mola, A. (1917). Medios sociales preventivos de la tuberculosis infantil. Revista Médica del Uruguay, 116-.

Mola, A. (1923). Escuela al Aire Libre n.3: Funcionamiento. Revista Médica del Uruguay, 438-.

Mola, A. (1927). Escuelas al aire libre de Montevideo. Boletín del Instituto Internacional Americano de Protección a la Infancia, 1 (2), 199- 220.

Mola, A. (1928). The open-air schools of Montevideo. Bulletin of the Pan American Union, 62 (9), 572-585.

Mola, A. (1930a). Escuelas al aire libre de Montevideo. Boletin del Instituto Internacional Americano de Protección a la Infancia, 3 (4), 707-733.

Mola, A. (1930b). Escuelas al aire libre de Montevideo. Montevideo: Imprenta Nacional.

Mola, A. (1936). Tercer Congreso Internacional de Escuelas al Aire Libre. Boletín del Instituto Internacional Americano de Protección a la Infancia, 10 (1), 402-412.

Mola, A. (1940). Obras escolares al aire libre. Enciclopedia de Educación, 3/2 (1), 119-128.

Nunes, E. S. N. (2011). A infância como portadora do futuro: América Latina, 1916-1948. (Doctorado en Historia Social). Universidade de São Paulo.

Petrillo, L. M. (1932). Tratamiento general de la tuberculosis pulmonar infantil. Boletín del Instituto Internacional Americano de Protección a la Infancia, 6 (1), 63-81.

Rauch, A. (1983). Le souci du corps: histoire de l'hygiène en éducation physique. Paris: Press Universitaire de France.

Rodrigo, C. R. y Lis, I. P. (1999). Higienismo, educación ambiental y previsión escolar: antecedentes y prácticas de educación social en Espanha (1900-1936). València: Universitat de València.

Rodríguez, I. T. (2015). Orden y naturaleza en la escuela al aire libre: el colegio para la institucio\#n Teresiana en Alicante de Rafael de La Hoz y Gerardo Olivares. (Doctorado en Arquitectura). Universidad Politécnica de Madrid.

Rodríguez, S. R. (1909). Proyecto instituyendo los paseos y excursiones escolares en las escuelas públicas. En Cuerpo Médico Escolar Nacional. (1910). La higiene escolar en la República Oriental del Uruguay: trabajo presentado al III Congreso Internacional de Higiene Escolar - Paris 1910. Montevideo: A. Barreiro y Ramos.

Rodríguez, S. R. (1914). Escuela al aire libre. En Cuerpo Médico Escolar Nacional. Memoria correspondiente al año 1913. Montevideo: El Siglo Ilustrado.

Rogé, E. (1910). Las escuelas al aire libre ó selváticas. Anales de Instrucción Primaria, 8 (1-6), 151-157.

Ruchat, M. (2003). Jean Dupertuis (1886-1951). En A. Châtelet, D. Lerch y J. Luc (Ed.), L'école de plein air: une expérience pédagogique et architecturale dans l'Europe du XXe siècle (pp. 261-270). Paris: Éditions Recherches.

Savoye, A. (2003). Écoles de plein air et educatión nouvelle en France (1920-1950) 2003 En A. Châtelet, D. Lerch y J. Luc (Ed.), L'école de plein air: une expérience pédagogique et architecturale dans l'Europe du XXe siècle (pp. 280-288). Paris: Éditions Recherches.

Schiaffino, R. (1914). Morbilidad y profilaxis de los escolares. En Cuerpo Médico Escolar Nacional. Memoria correspondiente al año 1913. Montevideo: El Siglo Ilustrado.

Schiaffino, R. (1929). Organización médico-escolar en el Uruguay. Boletín del Instituto Internacional Americano de Protección a la Infancia, 3 (1), 99-123.

Sirost, O. (2009). La vie au grand air ou l'invention occidentale des milieux récréatifs. En O. Sirost (Ed.), La vie au grand air: aventures du corps et évasions vers la nature ( $\mathrm{pp} 7-44)$. Nancy: Presses Universitaires.

Soares, C. L. (2016). Uma educação pela natureza: a vida ao ar libre, o corpo e a ordem urbana. Campinas: Autores Associados.

Turnes, A. L. (2015). La Sociedad Uruguaya de Pediatria en su centenario, 1915-2015. Montevideo: SUP.

Villaret, S. (2005). Histoire du naturisme en France depuis le siècle des lumières. Paris: Vuibert.

Villaret, S. y Saint-Martin, J.-P. (2004). Écoles de plein air et naturisme : une innovation en milieu scolaire (1887-1935). Mouvement \& Sport Sciences, 51, 11-28. 


\section{Notas}

1 Creada en 1889, la Comisión Nacional de Caridad y Beneficencia Pública era responsable por un hospital y tres orfanatos. En 1908 empezó la primera iniciativa de colonia de vacaciones de Uruguay.

2 Las dos primeras escuelas al aire libre argentinas fueran creadas en 1909 en parques de Buenos Aires.

3 La Liga Uruguaya contra la Tuberculosis fue creada en 1902 y pasó a responder a la Asistencia Pública Nacional en 1913. Mantuve, sin embargo, cierta autonomía, recibiendo subsidio del Comité de Damas.

4 En el año de 1928 un proyecto para la creación de una colonia marítima y de un preventorio infantil fue elaborado por consejeros del Consejo de Enseñanza Primaria y Normal (CEPN) - Eduardo Jiménez de Aréchaga, Emilio Oribe y Emilio Verdesio. La colonia marítima empezó sus actividades en el inicio del año de 1929. Los documentos divergen sobre el local de su instalación, si la playa de Buceo o de Malvin. Su designación también oscilaba entre colonia marítima, escuela marítima y colonia marítima escolar. El preventorio fue creado en 1930 en Colón, siendo por veces denominado como Escuela al Aire Libre 'Preventorium'. Las dos instituciones mantuvieran relaciones con la Comisión Especial de Campamentos Escolares del CEPN y la colonia marítima tuvo como médico inspector Manuel Landera (Dirección, 1929; Petrillo, 1932; Schiaffino, 1929).

5 Américo Mola cursó los estudios superiores en la Facultad de Medicina de Montevideo, trabajó en el Cuerpo Médico Escolar y fue el responsable por la inspección médica de las tres escuelas al aire libre creadas en Montevideo. En los textos que publicó no hizo referencia a la colonia marítima ni al preventorio infantil, probablemente por que no era su médico inspector responsable (Mola, 1930a, 1930b). A lo largo de su carrera fue socio de la Sociedad de Pediatría de Montevideo (uno de sus fundadores) y miembro de la Sociedad de Pediatría de Paris, así como presidente del Comité Internacional de Escuelas al Aire Libre (Turnes, 2015).

6 Rollier empleó la helioterapia para prevenir y curar la tuberculosis en sus instituciones médico-educativas en Suiza. Su nombre y sus métodos de tratamiento fueran reconocidos mundialmente debido a la amplia circulación de sus libros y de postales de sus instituciones (Woloshyn, 2013).

7 A propósito del Instituto Internacional Americano de Protección a la Infancia (actual Instituto Interamericano del Niño, la Niña y Adolescentes), de sus Congresos Panamericanos del Niño y boletines, conferir las investigaciones de Camarillo (2014) y Nunes (2011).

8 La invitación para que Morquio presidiese el Congreso Internacional de Protección a la Infancia resultaba del hecho de que él presidía el IIAPI, fundado oficialmente en 1927 (Mariano y Mariano, 2016).

9 Entre los años de 1927 y 1928 diversos artículos sobre escuelas al aire libre, campamentos y colonias de vacaciones fueran traducidos y publicados en la Enciclopedia de Educación. En este mismo momento fue presentado por Eduardo Jiménez de Aréchaga, Emilio Oribe y Emilio Verdesio el proyecto de la colonia marítima y del preventorio infantil (Dirección, 1929; Schiaffino, 1929). En 1927 el profesor Carlos Vaz Ferreira también presentó su antiguo y ambicioso proyecto de parque escolar, institución al aire libre que preveía servir a todos los escolares de Montevideo (Carbonell, 1927; Ferreira, 1927; Ministerio, 1928).

10 Entre los cuales Bélgica, Chile, China, Egipto, Ecuador, España, Estonia, Estados Unidos, Francia, Grecia, Holanda, Hungría, Irán, Italia, Luxemburgo, Noruega, Perú, Polonia, Rumania, Suecia, Suiza e Uruguay (Brun, 1936).

11 Las escuelas al aire libre en Uruguay fueron convertidas y resignificadas para las escuelas de tiempo completo en el año 1989 (Martinis, 2013). 